\title{
Lattice QCD at maximal twist
}

\author{
R. Frezzotti ${ }^{\mathrm{a}}$ and G.C. Rossi ${ }^{\mathrm{a} * \dagger}$ \\ ${ }^{a}$ Dipartimento di Fisica, Università di Roma Tor Vergata and INFN Sezione di Tor Vergata \\ Via della Ricerca Scientifica - 00133 Roma (ITALY)
}

In this review we discuss the general features of maximally twisted lattice QCD. In particular, we illustrate how automatic $\mathrm{O}(a)$ improvement can be achieved and how it is possible to set up a lattice regularization scheme where the problem of wrong chirality mixing (be it finite or infinite) affecting the computation of the matrix elements of the $\mathcal{C} \mathcal{P}$-conserving effective weak Hamiltonian is neatly avoided, while having at the same time a positive determinant even for non-degenerate quark pairs. The question of reducing the large cutoff effects that appear when the quark mass tends to zero as a consequence of parity and iso-spin breaking in the action is also addressed. It is shown that such dangerous lattice artifacts are strongly suppressed if the clover term is added to the action or, alternatively, the critical mass is chosen so as to enforce the restoration of parity.

\section{Introduction and content}

Waiting for new generation computers that might allow full-fledged simulations with exactly chirally invariant fermions, i.e. fermions obeying the Ginsparg-Wilson condition [1], a viable alternative could be to use maximally twisted Wilson fermions [2 34 516], possibly coupled with a judicious choice of the gauge action [7].

In this review we wish to outline the structure and the properties of maximally twisted lattice QCD (Mtm-LQCD) as developed in refs. 3456. Soon after introducing the idea that to avoid exceptional configurations in Wilson fermion simulations one should introduce quarks in flavour pairs and have the Wilson term rotated with respect to the quark mass term by an axial rotation in iso-spin space, it was realized that an especially useful choice for that angle is to set it at its maximal value, $|\omega|=\pi / 2$, because in this situation $\mathrm{O}(a)$ (actually $\left.\mathrm{O}\left(a^{2 k+1}\right), k \geq 0\right)$ improvement of physical quantities is automatic (sect. 2).

It was then shown in 4 that the nice improvement properties enjoyed by Mtm-LQCD, derived for pairs of mass degenerate quarks in [3], can be immediately extended to the more interest-

\footnotetext{
*Speaker

${ }^{\dagger}$ G.C.R. wishes to thank NIC at Desy-Zeuthen and the Humboldt Foundation for financial support.
}

ing non-degenerate case, without loosing the positivity of the corresponding fermion determinant (sect. 3). This last property is obviously crucial if one wants to be able to set up workable Monte Carlo-like simulation algorithms for QCD.

With these ingredients and exploiting the flexibility offered by the possibility of regularizing different valence flavours with different value of the Wilson parameter, $r$, it is possible to construct [4] a hybrid theory, where sea quarks are introduced as pairs of non-degenerate particles and valence quarks are regularized as Österwalder-Seiler [8] fermions, such that no "wrong chirality" mixing [9] affects the computation of the matrix elements of the $\mathcal{C} \mathcal{P}$-conserving $\Delta S=1,2$ effective weak Hamiltonian (sect. 4).

Despite all these nice features, there remain important cutoff effects, originating from the breaking of parity and iso-spin induced by the presence of the twisted Wilson term in the action, which tend to become large as the quark mass is lowered. These lattice artifacts have been discussed both in chiral perturbation theory $(\chi \mathrm{PT})[10-[13$, as well as in the language of the Symanzik expansion [6] with the conclusion that they can be substantially reduced if the clover term [14 is introduced in the action with its non-perturbatively fixed $c_{S W}$ coefficient [15] or, alternatively, if the critical mass is chosen in some "optimal way" (sect. 515). 
For lack of space we will not discuss how and to what extent the strategy outlined above for improvement can be extended to the Schrödinger functional formalism 1617. Nor we will address the very important issue of meta-stabilities 18 1920, that are seen to affect unquenched simulations 21] at coarse lattice spacing, and the closely related flavour breaking effects visible in the value of the square mass difference between charged and neutral pions 22. These questions and the the present status of quenched and unquenched twisted simulations have been recently reviewed in ref. 23. In the concluding section (sect. 61) we will only offer some hints on possible ways to overcome (even on coarse lattice spacings) difficulties associated with the existence of metastable phases and flavour breaking effects.

\section{Improvement}

The twisted fermion action for a pair of degenerate quarks in the "physical basis" (where the parameter which gives a non-vanishing mass to pions, $m_{q}$, is real) has the expression

$S_{\mathrm{F}}^{\omega}=a^{4} \sum_{x} \bar{\psi}(x)\left[\gamma \tilde{\nabla}+W_{\mathrm{cr}} e^{-i \omega \gamma_{5} \tau_{3}}+m_{q}\right] \psi(x)$.

In eq. (11) we have introduced the following definitions: $\gamma \tilde{\nabla}=\frac{1}{2} \gamma_{\mu}\left(\nabla_{\mu}^{\star}+\nabla_{\mu}\right)$ with $\nabla_{\mu}$ and $\nabla_{\mu}^{\star}$ the forward and backward lattice covariant derivatives, respectively, and $W_{\mathrm{cr}}=-r \frac{a}{2} \nabla_{\mu}^{\star} \nabla_{\mu}+M_{\mathrm{cr}}(r)$ with $M_{\mathrm{cr}}(r)$ the critical mass. We observe that to match the $r$-parity property of the Wilson term $M_{\mathrm{cr}}(r)$ is necessarily an odd function of $r$. The form of the fermionic action in the "twisted basis" is obtained by performing on the fermion fields the axial rotation

$\psi \rightarrow e^{i \omega \gamma_{5} \tau_{3} / 2} \psi, \quad \bar{\psi} \rightarrow \bar{\psi} e^{i \omega \gamma_{5} \tau_{3} / 2}$.

The first observation about $S_{\mathrm{F}}^{\omega}$ (and the one that originally led to its introduction) is that the determinant of the associated Dirac-Wilson operator is positive definite for any $\omega \neq 0$ and $m_{q} \neq 0$ as its expression is seen to be given by

$$
\begin{aligned}
& \mathcal{D}_{F}^{\omega}=\operatorname{det}\left[\left(D_{\mathrm{W}}^{\mathrm{cr}}+m_{q} \cos \omega\right)^{\dagger}\left(D_{\mathrm{W}}^{\mathrm{cr}}+m_{q} \cos \omega\right)+\right. \\
& \left.+m_{q}^{2} \sin ^{2} \omega\right], \quad D_{\mathrm{W}}^{\mathrm{cr}}=\gamma \tilde{\nabla}+W_{\mathrm{cr}}
\end{aligned}
$$

The lattice action (11) is invariant under the (spurionic) transformations

1) $\mathcal{R}_{5} \times(r \rightarrow-r) \times\left(m_{q} \rightarrow-m_{q}\right)$, where

$\mathcal{R}_{5}:\left\{\begin{array}{ll}\psi & \rightarrow \psi^{\prime}=\gamma_{5} \psi \\ \bar{\psi} & \rightarrow \quad \bar{\psi}^{\prime}=-\bar{\psi} \gamma_{5}\end{array} \quad \mathcal{R}_{5}^{2}=1\right.$

2) $\mathcal{D}_{d} \times(r \rightarrow-r) \times\left(m_{q} \rightarrow-m_{q}\right)$, with

$\mathcal{D}_{d}:\left\{\begin{array}{l}\left.U_{\mu}(x) \rightarrow U_{\mu}^{\dagger}(-x-a \hat{\mu})\right) \\ (\psi(x), \bar{\psi}(x)) \rightarrow e^{3 i \pi / 2}(\psi(-x), \bar{\psi}(-x))\end{array}\right.$

These invariances imply for the lattice vacuum expectation values (v.e.v.'s) of (multi-local) operators, $O(x)=O\left(x_{1}, x_{2}, \ldots, x_{n}\right), x_{1} \neq x_{2} \neq \ldots \neq$ $x_{n}$, the relations

1) $\left.\langle O(x)\rangle\right|_{\left(r, m_{q}\right)} ^{(\omega)}=\left.(-1)^{P_{\mathcal{R}_{5}}[O]}\langle O(x)\rangle\right|_{\left(-r,-m_{q}\right)} ^{(\omega)}$
2) $\left.\langle O(x)\rangle\right|_{\left(r, m_{q}\right)} ^{(\omega)}=\left.(-1)^{d[O]}\langle O(-x)\rangle\right|_{\left(-r,-m_{q}\right)} ^{(\omega)}$,

where $P_{\mathcal{R}_{5}[O]}$ is the $\mathcal{R}_{5}$-parity of $O$ and $d[O]$ its mass dimension.

We now prove that i) for generic values of the twisting angle, $\omega$, averages (Wilson average - WA) of v.e.v.'s computed with opposite values of $r$ are free of $\mathrm{O}\left(a^{2 k+1}\right), k \geq 0$, cutoff effects; ii) for the special value $|\omega|=\pi / 2$ averaging is unnecessary.

These results follow from using the Symanzik idea 24 that lattice artifacts can be described in terms of the continuum correlators of an effective low energy theory renormalized at the scale $a^{-1}$, as well as the symmetry properties of the lattice theory and the corresponding ones enjoyed by the associated continuum theory.

i) $|\omega| \neq \pi / 2$ - We write the Symanzik expansion of the lattice v.e.v. of a multi-local, multiplicatively renormalizable (m.r.) operator in the schematic form

$$
\begin{aligned}
& \left.\langle O(x)\rangle\right|_{\left(r, m_{q}\right)} ^{(\omega)}=\left[\zeta_{O}^{O}(\omega, r)\langle O(x)\rangle+\right. \\
& \left.+a \sum_{\ell} \eta_{O_{\ell}}^{O}(\omega, r)\left\langle O_{\ell}(x)\right\rangle\right]_{\left(m_{q}\right)}^{\mathrm{cont}}+\mathrm{O}\left(a^{2}\right),
\end{aligned}
$$

where the operators $O_{\ell}$ have mass dimension of one unit larger than that of $O, d\left[O_{\ell}\right]=d[O]+1$. We explicitly remark that factors of $m_{q}$ may appear 
in $O_{\ell}$. The lattice invariance (7) allow us to write

$$
\begin{aligned}
& \left.\langle O(x)\rangle\right|_{\left(r, m_{q}\right)} ^{(\omega)}=\left.(-1)^{d[O]}\langle O(-x)\rangle\right|_{\left(-r,-m_{q}\right)} ^{(\omega)}= \\
& =(-1)^{d[O]\left[\zeta_{O}^{O}(\omega,-r)\langle O(-x)\rangle+\right.} \\
& \left.+a \sum_{\ell} \eta_{O_{\ell}}^{O}(\omega,-r)\left\langle O_{\ell}(-x)\right\rangle\right]_{\left(-m_{q}\right)}^{\mathrm{cont}}+\mathrm{O}\left(a^{2}\right) .
\end{aligned}
$$

We can now use the invariance $\mathcal{D}_{d} \times\left(m_{q} \rightarrow-m_{q}\right)$ of the continuum theory to bring continuum correlators computed in $-x$ and $-m_{q}$ to correlators computed in $x$ and $m_{q}$. One thus gets

$$
\begin{aligned}
& \left.\langle O(x)\rangle\right|_{\left(r, m_{q}\right)} ^{(\omega)}=\left[\zeta_{O}^{O}(\omega,-r)\langle O(x)\rangle+\right. \\
& \left.-a \sum_{\ell} \eta_{O_{\ell}}^{O}(\omega,-r)\left\langle O_{\ell}(x)\right\rangle\right]_{\left(m_{q}\right)}^{\mathrm{cont}}+\mathrm{O}\left(a^{2}\right) .
\end{aligned}
$$

Comparison with (8) gives the relations

$\zeta_{O}^{O}(\omega, r)=\zeta_{O}^{O}(\omega,-r), \eta_{O_{\ell}}^{O}(\omega, r)=-\eta_{O_{\ell}}^{O}(\omega,-r)$, which imply the WA improvement formula

$$
\begin{aligned}
& \left.\langle O(x)\rangle\right|_{\left(r, m_{q}\right)} ^{(\omega)}+\left.\langle O(x)\rangle\right|_{\left(-r, m_{q}\right)} ^{(\omega)}= \\
& =\left.2 \zeta_{O}^{O}(\omega, r)\langle O(x)\rangle\right|_{\left(m_{q}\right)} ^{\mathrm{cont}}+\mathrm{O}\left(a^{2}\right) .
\end{aligned}
$$

Exploiting the $\mathcal{R}_{5}$-parity transformation properties of $O$, the previous formula can be rewritten in the form of a mass average (MA)

$$
\begin{aligned}
& \left.\langle O(x)\rangle\right|_{\left(r, m_{q}\right)} ^{(\omega)}+\left.(-1)^{P_{\mathcal{R}_{5}}[O]}\langle O(x)\rangle\right|_{\left(r,-m_{q}\right)} ^{(\omega)}= \\
& =\left.2 \zeta_{O}^{O}(\omega, r)\langle O(x)\rangle\right|_{\left(m_{q}\right)} ^{\mathrm{cont}}+\mathrm{O}\left(a^{2}\right) .
\end{aligned}
$$

Note that in the presence of spontaneous chiral symmetry breaking the two terms in the l.h.s. of (12) will be in general different even in the limit $m_{q} \rightarrow 0^{3}$.

One can easily convince oneself that the whole argument developed above is actually valid for any odd powers of $a$ in the Symanzik expansion. As a result all $\mathrm{O}\left(a^{2 k+1}\right), k \geq 0$, terms get cancel

\footnotetext{
${ }^{3}$ In the absence of spontaneous chiral symmetry breaking, like in 2-dimensions, lattice data show improvement at any $\omega$ even without WA 25$]$.
}

by taking either the Wilson or the mass average of lattice correlators.

ii) $|\omega|=\pi / 2$ - At maximal twist the lattice action is invariant under the further transformation (which does not require changing sign to $r$ ) $\mathcal{P} \times \mathcal{D}_{d} \times\left(m_{q} \rightarrow-m_{q}\right)$, where $\mathcal{P}$ is ordinary parity $\left(x_{P}=\left(x_{0},-\mathbf{x}\right)\right)$

$\mathcal{P}:\left\{\begin{array}{l}\psi(x) \rightarrow \gamma_{0} \psi\left(x_{P}\right), \quad \bar{\psi}(x) \rightarrow \bar{\psi}\left(x_{P}\right) \gamma_{0} \\ U_{0}(x) \rightarrow U_{0}\left(x_{P}\right), \\ U_{k}(x) \rightarrow U_{k}^{\dagger}\left(x_{P}-a \hat{k}\right), \quad k=1,2,3\end{array}\right.$

This fact allows to prove that (for parity-even operators) $\mathrm{O}\left(a^{2 k+1}\right), k \geq 0$, improvement is automatic. The proof (see Appendix of ref. 6] ) closely follows the previous line of reasoning and does not depend on the $r$-parity of $M_{\mathrm{cr}}$. One starts with eq. (8) taken at, say, $\omega=\pi / 2$. Using the lattice symmetry $\mathcal{P} \times \mathcal{D}_{d} \times\left(m_{q} \rightarrow-m_{q}\right)$, it yields

$\left.\langle O(x)\rangle\right|_{\left(r, m_{q}\right)} ^{\pi / 2}=\left.(-1)^{P[O]+d[O]}\left\langle O\left(-x_{P}\right)\right\rangle\right|_{\left(r,-m_{q}\right)} ^{\pi / 2}=$
$=(-1)^{P[O]+d[O]\left[\zeta_{O}^{O}(r)\left\langle O\left(-x_{P}\right)\right\rangle+\right.}$
$\left.+a \sum_{\ell} \eta_{O_{\ell}}^{O}(r)\left\langle O_{\ell}\left(-x_{P}\right)\right\rangle\right]_{\left(-m_{q}\right)}^{\mathrm{cont}}+\mathrm{O}\left(a^{2}\right)$

In eq. (14) $P[O]$ is the parity of $O$. As before, we can act with the corresponding continuum symmetry transformation on the r.h.s. of this equation to bring back its space-time argument from $-x_{P}$ to $x$, obtaining

$\left.\langle O(x)\rangle\right|_{\left(r, m_{q}\right)} ^{\pi / 2}=\left[\zeta_{O}^{O}(r)\langle O(x)\rangle+\right.$
$\left.-a \sum_{\ell} \eta_{O_{\ell}}^{O}(r)(-1)^{P\left[O_{\ell}\right]+P[O]}\left\langle O_{\ell}(x)\right\rangle\right]_{\left(m_{q}\right)}^{\mathrm{cont}}+\mathrm{O}\left(a^{2}\right)$.

Comparing now eq. (14) with eq. (15) we get

$-(-1)^{P\left[O_{\ell}\right]+P[O]} \eta_{O_{\ell}}^{O}(r)=\eta_{O_{\ell}}^{O}(r)$,

which tells us that, if $O$ is a parity-even operator $(P[O]=0 \bmod (2))$, all $\mathrm{O}\left(a^{2 k+1}\right)(k \geq 0)$ terms in the Symanzik expansion of $\left.\langle O(x)\rangle\right|_{\left(r, m_{q}\right)} ^{\pi / 2}$ are multiplied by continuum matrix elements of parity-odd operators that hence vanish owing to the parity invariance of the continuum theory. 


\section{Non-degenerate quarks}

In this section we want to show that the nice properties of Mtm-LQCD, i.e. automatic improvement and positivity of the fermion determinant, established for the mass degenerate case, can be extended to the non-degenerate case.

It was proved in ref. 4 that the action

$$
\begin{aligned}
& S_{\mathrm{F}, n d}[\psi, \bar{\psi}, U]= \\
& =a^{4} \sum_{x} \bar{\psi}(x)\left[\gamma \widetilde{\nabla}-i \gamma_{5} \tau_{1} W_{\mathrm{cr}}+m_{q}+\tau_{3} \epsilon_{q}\right] \psi(x)
\end{aligned}
$$

describes the (lattice regularized) gauge interactions of a pair of non-degenerate quarks with renormalized masses $\hat{m}_{q}^{( \pm)}=Z_{P}^{-1} m_{q} \pm Z_{S}^{-1} \epsilon_{q}$, where $Z_{P}$ and $Z_{S}$ are the renormalization constants of the pseudo-scalar and scalar quark density, computed in the massless standard Wilson theory. These results follow 4 from the analysis of the chiral WTI's associated to the action (17).

We now prove i) automatic improvement and ii) positivity of the determinant of the DiracWilson operator in (17).

i) Automatic improvement of v.e.v.'s of parityeven operators follows from the invariance of (17) under the transformation $\mathcal{P} \times \mathcal{D}_{d} \times\left(m_{q} \rightarrow-m_{q}\right) \times$ $\left(\epsilon_{q} \rightarrow-\epsilon_{q}\right)$ by an argument which exactly parallels the one developed in sect. 2 for the maximally twisted case.

ii) Positivity of

$$
\operatorname{det}\left[D_{n d}\right]=\operatorname{det}\left[\gamma \widetilde{\nabla}-i \gamma_{5} \tau_{1} W_{\text {cr }}+m_{q}+\tau_{3} \epsilon_{q}\right]
$$

is proved by first noticing that $\operatorname{det}\left[D_{n d}\right]$ is even in $m_{q}$ and $\epsilon_{q}$ as it follows from the chain of equalities

$$
\begin{aligned}
& \operatorname{det}\left[D_{n d}\right]=\operatorname{det}\left[\gamma_{5} e^{i \pi \gamma_{5} \tau_{1} / 4} D_{n d} e^{i \pi \gamma_{5} \tau_{1} / 4}\right]= \\
& =\operatorname{det}\left[Q_{\mathrm{cr}}+i \tau_{1} m_{q}+\gamma_{5} \tau_{3} \epsilon_{q}\right]= \\
& =\operatorname{det}\left[\begin{array}{cc}
Q_{\mathrm{cr}}+\epsilon_{q} \gamma_{5} & i m_{q} \\
i m_{q} & Q_{\mathrm{cr}}-\epsilon_{q} \gamma_{5}
\end{array}\right]
\end{aligned}
$$

where we have defined $Q_{\mathrm{cr}}=\gamma_{5}\left[\gamma \tilde{\nabla}+W_{\mathrm{cr}}\right]=Q_{\mathrm{cr}}^{\dagger}$. To proceed it is convenient to further write

$\operatorname{det}\left[D_{n d}\right]=\operatorname{det}\left[Q_{\mathrm{cr}}^{2}+m_{q}^{2}-\epsilon_{q}^{2}\right] \operatorname{det}\left[1+2 \epsilon_{q} B\right],(20)$

$B=X \gamma \tilde{\nabla} X=-B^{\dagger}, X=\left(Q_{\mathrm{cr}}^{2}+m_{q}^{2}-\epsilon_{q}^{2}\right)^{-1 / 2}$.

If $m_{q}^{2}-\epsilon_{q}^{2}>0$, the first factor in the r.h.s. of (20) is positive. Expanding in $\epsilon_{q} B$, we get for the second $\log \operatorname{det}\left[1+2 \epsilon_{q} B\right]=\operatorname{tr}\left[\log \left(1+2 \epsilon_{q} B\right)\right]=$

$$
\begin{aligned}
& =-\sum_{k=1}^{\infty} \frac{\left(-2 \epsilon_{q}\right)^{k}}{k} \operatorname{tr}\left[B^{k}\right]=-\sum_{n=1}^{\infty} \frac{\left(4 \epsilon_{q}^{2}\right)^{n}}{2 n} \operatorname{tr}\left[\left(-B^{\dagger} B\right)^{n}\right]= \\
& =\frac{1}{2} \operatorname{tr}\left[\log \left(1+4 \epsilon_{q}^{2} B^{\dagger} B\right)\right] \geq 0 .
\end{aligned}
$$

In the second equality we have used evenness in $\epsilon_{q}$ and the anti-hermiticity of $B$. The nonnegativeness of (21) is evident as the argument of the last logarithm is an operator with a norm certainly not smaller than unit.

We conclude with the following important observation. We have proved the positivity (rather the non-negativity) of the determinant (19) of the mass non-degenerate Wilson-Dirac operator under the assumption that the bare parameters $m_{q}$ and $\epsilon_{q}$ satisfy the inequality $m_{q}^{2}-\epsilon_{q}^{2}>0$. For the renormalized masses of any sea doublet this bound implies $\frac{Z_{P}}{Z_{S}}>\frac{\hat{m}_{q}^{(+)}-\hat{m}_{q}^{(-)}}{\hat{m}_{q}^{(+)}+\hat{m}_{q}^{(-)}}$. Whether or not such a limitation can be a real problem for actual simulations is to be seen. We note that in the worst case, taking $\hat{m}_{q}^{(+)}=\hat{m}_{c} \sim 1200 \mathrm{MeV}$ and $\hat{m}_{q}^{(-)}=\hat{m}_{s} \sim 100 \mathrm{MeV}$, one gets $\frac{Z_{P}}{Z_{S}}>0.85$.

\section{Weak matrix elements}

The complicated pattern of "wrong chirality" mixing affecting the construction of the renormalizable effective weak Hamiltonian operator on the lattice when Wilson fermions are employed 926 has up to now prevented a reliable, full-fledged evaluation of the phenomenologically important $\mathcal{C P}$-conserving weak matrix elements ${ }^{4}$ relevant for the evaluation of $B_{K}$ and the amplitudes appearing in the famous $\Delta I=1 / 2$ rule 3031 .

Mtm-LQCD offers a comparatively simple framework where the problem of "wrong chirality" mixing can be neatly circumvented leading to a cheap computational scheme. As an extra bonus automatic improvement, which we discussed in the previous sections, is guaranteed [5].

The idea is to make recourse to a hybrid formulation in which sea quarks are introduced as maximally twisted mass non-degenerate pairs, while valence quarks are taken as Österwalder-Seiler (OS) flavour singlet fermions. This formulation

${ }^{4}$ See, however, ref. [27] for some alternative way-outs if the Wilson theory is fully $\mathrm{O}(a)$ improved 2829 . 
can be made fully local 32 if for each valence quark a ghost field is introduced with the purpose of canceling the determinant coming form the valence quark integration. Provided sea and valence quarks are given the same renormalized masses, the sector of the theory where only correlators with no ghost fields are considered is unitary, with "partial quenching" effects due a slightly different sea and valence quarks regularization starting only from order $a^{2}$ 33.

The key point on which the rest of this section is focused is the fact that killing the effects of "wrong chirality" mixing in the lattice data of weak matrix elements requires to consider a specific regularization of the valence sector of the theory which depends on the particular matrix element one is willing to compute. In particular we will see that it may be necessary to replicate certain valence flavours assigning appropriately tuned signs to the corresponding Wilson terms.

Notice that in the Mtm-LQCD hybrid formulation we are considering parity and flavour breaking effects can only arise from the valence sector. The flavour symmetry of valence sector can be larger than the direct product of $N_{f} \mathrm{U}(1)$-factors, depending on the choice of the values of the Wilson parameters.

\section{1. $B_{K}$ with no mixing}

In the formal continuum QCD theory with 4 flavours (QCD4) $B_{K}$ is defined by the formula ${ }^{5}$

$\left\langle\bar{K}_{0}\left|\hat{\mathcal{O}}_{V V+A A}^{\Delta S=2}(\mu)\right| K_{0}\right\rangle=\frac{16}{3} M_{K}^{2} F_{K}^{2} B_{K}(\mu)$

$\mathcal{O}_{V V+A A}^{\Delta S=2}=\left(\bar{s} \gamma_{\mu} d\right)\left(\bar{s} \gamma_{\mu} d\right)+\left(\bar{s} \gamma_{\mu} \gamma_{5} d\right)\left(\bar{s} \gamma_{\mu} \gamma_{5} d\right)$

and can be extracted e.g. from the correlator

$C_{K \mathcal{O} K}=\left\langle\Phi_{K}(x) \mathcal{O}_{V V+A A}^{\Delta S=2}(0) \Phi_{K}(y)\right\rangle$,

$\Phi_{K}=\bar{d} \gamma_{5} s$

The key observation of ref. [5] is that the same information can be extracted from the correlator

$C_{K^{\prime} \mathcal{Q} K}=\left\langle\Phi_{K_{v}^{\prime}}(x) 2 \mathcal{Q}_{V V+A A}^{\Delta S=2}(0) \Phi_{K_{v}}(y)\right\rangle$,

$\Phi_{K_{v}^{\prime}}=\bar{d}_{v}^{\prime} \gamma_{5} s_{v}^{\prime}, \quad \Phi_{K_{v}}=\bar{d}_{v} \gamma_{5} s_{v}$,

\footnotetext{
${ }^{5}$ Undefined notations as well as further details on the renormalization properties of the different regularizations we will discuss below can be found in ref. [5] and will not be repeated here for lack of space.
}

$\mathcal{Q}_{V V+A A}^{\Delta S=2}=$

$=\left(\bar{s}_{v} \gamma_{\mu} d_{v}\right)\left(\bar{s}_{v}^{\prime} \gamma_{\mu} d_{v}^{\prime}\right)+\left(\bar{s}_{v} \gamma_{\mu} \gamma_{5} d_{v}\right)\left(\bar{s}_{v}^{\prime} \gamma_{\mu} \gamma_{5} d_{v}^{\prime}\right)+$

$+\left(\bar{s}_{v} \gamma_{\mu} d_{v}^{\prime}\right)\left(\bar{s}_{v}^{\prime} \gamma_{\mu} d_{v}\right)+\left(\bar{s}_{v} \gamma_{\mu} \gamma_{5} d_{v}^{\prime}\right)\left(\bar{s}_{v}^{\prime} \gamma_{\mu} \gamma_{5} d_{v}\right)$,

where $d_{v}^{\prime}$ and $s_{v}^{\prime}$ are replicas of $d_{v}$ and $s_{v}$ valence quarks with $m_{v}^{d^{\prime}, s^{\prime}}=m_{v}^{d, s}$. This statement can be proved by noting that, if QCD4 and the theory which for short we will call $4 \mathrm{~s} 6 \mathrm{v}$ (meaning 4 sea quarks - $u_{s}, d_{s}, s_{s}, c_{s}$ - and 6 valence quarks - $u_{v}$, $\left.u_{v}^{\prime} d_{v}, d_{v}^{\prime}, s_{v}, c_{v}\right)$ are regularized in the same way (say à la GW), the two correlators (23) and (25) are equal, simply because they give rise to exactly the same Wick contractions. This also implies that the renormalization constant of $\mathcal{Q}_{V V+A A}^{\Delta S}$ in $4 \mathrm{~s} 6 \mathrm{v}$ is equal to that of $\mathcal{O}_{V V+A A}^{\Delta S}$ in QCD4 (if the same renormalizalization condition is employed). Similarly $\bar{d}_{v} \gamma_{5} s_{v}$ and $\bar{d}_{v}^{\prime} \gamma_{5} s_{v}^{\prime}$ have the common renormalization constant equal to that of $\bar{d} \gamma_{5} s$.

It is not too difficult at this point to prove that, if we take sea and valence quarks of the same flavour to have the same renormalized masses and we regularize valence quarks as OS fermions having Wilson terms with $r_{v}^{d}=r_{v}^{s}=r_{v}^{d^{\prime}}=-r_{v}^{s^{\prime}}$, the matrix element (25) is not contaminated by unwanted "wrong chirality" mixing effects. In fact, the peculiar symmetry properties of the regularized action prevent the mixing of $\mathcal{Q}_{V V+A A}^{\Delta S=2}$ with all the other dimension 6 operators with $\Delta S=2$ that can be constructed with valence quarks.

The action of the regularized theory $4 \mathrm{~s} 6 \mathrm{v}^{L}$ constructed as described before is invariant (among others) under the following transformations

i) $\operatorname{Ex}\left(d_{v}, d_{v}^{\prime}\right) \times\left(m_{v}^{d} \leftrightarrow m_{v}^{d^{\prime}}\right)$

ii) $\operatorname{Ex}_{5}\left(s_{v}, s_{v}^{\prime}\right) \times\left(m_{v}^{s} \leftrightarrow-m_{v}^{s^{\prime}}\right)$

iii) $\mathcal{C} \times\left[\operatorname{Ex}\left(d_{v}, s_{v}\right) \times\left(m_{v}^{d} \leftrightarrow m_{v}^{s}\right)\right] \times$

$\times\left[\operatorname{Ex}_{5}\left(d_{v}^{\prime}, s_{v}^{\prime}\right) \times\left(m_{v}^{d^{\prime}} \leftrightarrow-m_{v}^{s^{\prime}}\right)\right]$

iv) $\mathcal{P}_{5} \times(M \rightarrow-M)$.

where ${ }^{6}$ ( $M$ is the whole set of mass parameter)

$\operatorname{Ex}\left(q_{v}^{f_{1}}, q_{v}^{f_{2}}\right): q_{v}^{f_{1}} \rightarrow q_{v}^{f_{2}} \quad \bar{q}_{v}^{f_{1}} \rightarrow \bar{q}_{v}^{f_{2}}$

$\operatorname{Ex}_{5}\left(q_{v}^{f_{1}}, q_{v}^{f_{2}}\right): \begin{cases}q_{v}^{f_{1}} \rightarrow \gamma_{5} q_{v}^{f_{2}} & \bar{q}_{v}^{f_{1}} \rightarrow-\bar{q}_{v}^{f_{2}} \gamma_{5} \\ q_{v}^{f_{2}} \rightarrow \gamma_{5} q_{v}^{f_{1}} & \bar{q}_{v}^{f_{2}} \rightarrow-\bar{q}_{v}^{f_{1}} \gamma_{5}\end{cases}$

$\mathcal{P}_{5}: \begin{cases}U \rightarrow \mathcal{P}[U] \\ q_{v}^{f}(x) \rightarrow \gamma_{5} \gamma_{0} q_{v}^{f}\left(x_{P}\right) & \bar{q}_{v}^{f}(x) \rightarrow-\bar{q}_{v}^{f}\left(x_{P}\right) \gamma_{0} \gamma_{5} \\ \psi^{j}(x) \rightarrow \gamma_{5} \gamma_{0} \psi^{j}\left(x_{P}\right) & \bar{\psi}^{j}(x) \rightarrow-\bar{\psi}^{j}\left(x_{P}\right) \gamma_{0} \gamma_{5}\end{cases}$

${ }^{6}$ To be precise these transformations must be appropriately extended to the ghost fields associated to each valence quark in order to make the full action invariant 5 . 
and $\mathcal{C}$ is charge conjugation. One checks that the operator $\mathcal{Q}_{V V+A A}^{\Delta S=2}$ is even under any of the above transformation, while the dimension 6 operators with which it can potentially mix (namely $\mathcal{Q}_{V V=}^{\Delta S=2}, \mathcal{Q}_{S S-P P}^{\Delta S=2}, \mathcal{Q}_{T T}^{\Delta S=2}, \mathcal{Q}_{S S+P P}^{\Delta S=2}, \mathcal{Q}_{V A \pm A V}^{\Delta S=2}$, $\mathcal{Q}_{S P \pm P S}^{\Delta S=2}$ and $\mathcal{Q}_{T \widetilde{T}}^{\Delta S}$ ) are all odd.

\subsection{Kaon decay amplitudes}

A long standing puzzle in low energy hadron physics, often referred to as "octet enhancement" or " $\Delta I=1 / 2$ rule", is represented by the surprisingly large experimental value of the ratio 30

$\mathrm{R}(K \rightarrow \pi \pi)=\frac{\left.\Gamma(K \rightarrow \pi \pi)\right|_{\Delta I=1 / 2}}{\left.\Gamma(K \rightarrow \pi \pi)\right|_{\Delta I=3 / 2}} \sim 400$.

Though the rate of the $\Delta I=3 / 2 K \rightarrow \pi \pi$ weak decays can be reasonably well computed within our present understanding of field theory (based on O.P.E. and renormalization group arguments), theoretical estimates of the $\Delta I=1 / 2$ amplitude give much too small values compared to the experimental number 34.

The lattice approach seems to be the natural framework where a first principle evaluation of such amplitides can be carried out. There are, however, severe difficulties in the process of establishing a proper strategy to accomplish this task. First of all, in Euclidean metric the procedure necessary to extract the kaon decay amplitudes of physical interest is complicated by IR subtleties arising from final (two-pion) state interactions 35. To attack this problem new interesting ideas have been recently put forward 3637 . Here we will only deal with the UV difficulties related to the construction of the renormalized effective weak Hamiltonian operator on the lattice and show how exploiting the flexiblity of the Mtm-LQCD formulation the problem of wrong chirality mixing can be brought to an amenable solution. For an interesting step forward in this direction see also 38 .

\subsubsection{Generalities}

In the Standard Model the decay of the $K$ meson into pions is described to leading order in the Fermi constant, $G_{\mathrm{F}}$, by the $\mathcal{C P}$-conserving, $\Delta S=1$ effective weak Hamiltonian, which (in the chiral limit) reads 30

$\mathcal{H}_{\text {eff }}^{\Delta S=1}=V_{u d} V_{u s}^{*} \frac{G_{\mathrm{F}}}{\sqrt{2}} \sum_{\ell= \pm} C_{\ell}\left(\mu / M_{\mathrm{W}}\right) \widehat{\mathcal{O}}^{\ell}(\mu)$,

with $V_{u d} V_{u s}^{*}$ the product of the appropriate elements of the CKM matrix ${ }^{7}$. The effective operator $\mathcal{H}_{\text {eff }}^{\Delta S=1}$ is obtained after having integrated out all degrees of freedom above some energy scale, $\Lambda$, with $\Lambda$ larger than the charm threshold, but still well below the $\mathrm{W}$-boson mass, $M_{\mathrm{W}}$ Consistently, the operators $\widehat{\mathcal{O}}^{ \pm}$in eq. (29) are renormalized at the scale $\mu$ with $m_{c} \ll \mu \ll M_{\mathrm{W}}$, while the Wilson coefficients $C_{ \pm}\left(\mu / M_{\mathrm{W}}\right)$ carry the information about the physics between $\mu$ and $M_{\mathrm{W}}$. The explicit expression of the bare operators corresponding to $\widehat{\mathcal{O}}^{ \pm}$is $\left(\gamma_{\mu}^{L}=\gamma_{\mu}\left(1-\gamma_{5}\right)\right)$

$2 \mathcal{O}^{ \pm}=\left[\left(\bar{s} \gamma_{\mu}^{L} u\right)\left(\bar{u} \gamma_{\mu}^{L} d\right) \pm\left(\bar{s} \gamma_{\mu}^{L} d\right)\left(\bar{u} \gamma_{\mu}^{L} u\right)\right]-[u \leftrightarrow c]$.

To make contact with experimental data it is enough to consider the decay of the neutral kaon, $K^{0}$, into either $\pi^{+} \pi^{-}$or $\pi^{0} \pi^{0}$ states 39404138 . Owing to the parity invariance of the formal continuum QCD4 theory, the relevant amplitudes $\mathcal{A}\left(K^{0} \rightarrow \pi^{+} \pi^{-}\right)$and $\mathcal{A}\left(K^{0} \rightarrow \pi^{0} \pi^{0}\right)$ can be written in terms of matrix elements of the renormalized parity odd operators, $\widehat{\mathcal{O}}_{V A}^{ \pm}(\mu)$, whose bare expression is

$$
\begin{aligned}
& 2 \mathcal{O}_{V A}^{ \pm}=\left[\left(\bar{s} \gamma_{\mu} u\right)\left(\bar{u} \gamma_{\mu} \gamma_{5} d\right) \pm\left(\bar{s} \gamma_{\mu} d\right)\left(\bar{u} \gamma_{\mu} \gamma_{5} u\right)+\right. \\
& \left.+\left(\bar{s} \gamma_{\mu} \gamma_{5} u\right)\left(\bar{u} \gamma_{\mu} d\right) \pm\left(\bar{s} \gamma_{\mu} \gamma_{5} d\right)\left(\bar{u} \gamma_{\mu} u\right)\right]-[u \leftrightarrow c] .
\end{aligned}
$$

\subsection{2. $K \rightarrow \pi \pi$ with no mixing}

The idea of the approach developed in ref. [5] is to extend the philosophy employed in sect. 4.1 for the computation of $B_{K}$ to the kaon decay amplitudes. In this case the required pattern of valence quark replicas will be somewhat more complicated and one needs to take four replicas of both $u_{v}$ and $c_{v}$ valence flavours. We immediately notice that this entails only a small extra computational burden (the evaluation of six rather than four valence quark propagators).

More precisely it can be shown that any information contained in the QCD4 correlator (meson charges are understood)

$C_{ \pm, K \pi \pi}^{\mathrm{QCD} 4}=\left.\left\langle\Phi_{\pi}(x) \Phi_{\pi}(z) \mathcal{O}_{V A}^{ \pm}(0) \Phi_{K}^{\dagger}(y)\right\rangle\right|^{\mathrm{QCD} 4}(30)$

${ }^{7}$ As usual, the top quark contribution, which is down by a factor $\mathrm{O}\left(V_{t d} V_{t s}^{\star} / V_{u d} V_{u s}^{\star} \simeq 10^{-3}\right)$, is neglected. 
can be recovered from the correlator

$$
C_{ \pm, K \pi \pi}^{4 s 10 v}=\left.\left\langle\Phi_{\pi}(x) \Phi_{\pi}(z) \mathcal{Q}_{V A}^{ \pm}(0) \Phi_{K}^{\dagger}(y)\right\rangle\right|^{4 s 10 v} .
$$

The model $4 s 10 v$ is intended as a theory where, besides the two doublets of sea quarks $(u, d)$ and $(s, c)$, in the valence sector the $u_{v}$ and $c_{v}$ flavours are replicated four times. Giving them the names $u_{v}^{[k]}$ and $c_{v}^{[k]}(k=0,1,2,3), \mathcal{Q}_{V A}^{ \pm}$must be taken to have the expression ${ }^{8}$

$\mathcal{Q}_{V A}^{ \pm}=\mathcal{Q}_{V A}^{ \pm[0]}+\mathcal{Q}_{V A}^{ \pm[1]}-\frac{1}{2} \mathcal{Q}_{V A}^{ \pm[2]}-\frac{1}{2} \mathcal{Q}_{V A}^{ \pm[3]}$,

$\mathcal{Q}_{V A}^{ \pm[k]}=\left.\mathcal{O}_{V A}^{ \pm}\right|_{u=u^{[k]}} ^{c=c^{[k]}}$.

We remark that the interpolating pion and kaon fields in eq. (31), besides the quarks $d$ and $s$, should be constructed with only the $u^{[0]}$ replica of the up quark. As in sect. 4.1 one can prove that, if the two theories QCD4 and $4 s 10 v$ are regularized in the same way, the correlators (30) and (31) are equal.

At this point the theory $4 s 10 v$ is regularized by taking the signs of the Wilson parameters of OS valence flavours to be in the particular relation

$$
\begin{aligned}
& r_{d}=r_{s}=r_{u^{[0]}}=-r_{u^{[1]}}=r_{u^{[2]}}=-r_{u^{[3]}}= \\
& =r_{c^{[0]}}=-r_{c^{[1]}}=r_{c^{[2]}}=-r_{c^{[3]}} .
\end{aligned}
$$

and the renormalized masses of (sea and valence) quarks of the same flavour are assigned the same value. We will give the name $4 s 10 v^{L}$ to this regularization of the $4 s 10 v$ theory.

One can prove that in $4 s 10 v^{L}$ the operators $\mathcal{Q}_{V A}^{ \pm}$are multiplicatively renormalizable, i.e. i) they mix neither with operators of dimension smaller than 6 , except for $\left(m_{c}^{2}-m_{u}^{2}\right)\left(m_{s}-\right.$ $\left.m_{d}\right) \bar{s} \gamma_{5} d$ which comes into play with a logarithmic divergent coefficient, ii) nor with operators of dimensions 6 .

i) We first prove that the pattern of mixing with operator of dimension $<6$ is as in the continuum, namely that the operators of dimension 3 and $5\left[\bar{s} \gamma_{5} d, \bar{s} \gamma_{5} \sigma \cdot F d\right]$ can only appear multiplied by the mass factors $\left(m_{c}^{2}-m_{u}^{2}\right)\left(m_{s}-m_{d}\right)$ and the operators $[\bar{s} d, \bar{s} \sigma \cdot F d]$ by $\left(m_{c}^{2}-m_{u}^{2}\right)\left(m_{s}^{2}-m_{d}^{2}\right)$. The various mass factors come from the symmetries enjoyed by the regularized theory $4 s 10 v^{L}$, according to the following scheme.

${ }^{8}$ From now on not to have much too clumsy notation we will drop the subscript $v$ on valence quark fields.
- $m_{c}-m_{u}$ from $\prod_{k} \operatorname{Ex}\left(u^{[k]}, c^{[k]}\right) \times\left(m_{u} \leftrightarrow m_{c}\right)$,

- $m_{s}-m_{d}$ from $\operatorname{Ex}(d, s) \times \mathcal{C} \times\left(m_{d} \leftrightarrow m_{s}\right)$,

- $m_{c}+m_{u}$ from $\left[\operatorname{Ex}_{5}\left(u^{[0]}, u^{[1]}\right) \times \operatorname{Ex}_{5}\left(u^{[2]}, u^{[3]}\right) \times\right.$ $\left.\times\left(m_{u} \rightarrow-m_{u}\right)\right] \times[u \rightarrow c]$,

- $m_{s}-m_{d}$ from $\mathcal{P}_{5} \times(M \rightarrow-M)$, but only in association with the operators $\bar{s} d$ and $\bar{s} \sigma \cdot F d$.

ii) Proving that the operators $\mathcal{Q}_{V A}^{ \pm}$do not mix with operators of dimension 6 can be done by setting all quark masses to zero $(M=0)$. Following [5], we proceed by showing that the symmetries of the regularized theory forbid the mixing of anyone of the four operators $\mathcal{Q}_{V A}^{ \pm[k]}$ appearing in eq. (32) with all the other operator of dimension 6 having the same unbroken quantum numbers. Let us start by discussing the case of $\mathcal{Q}_{V A}^{ \pm[0]}$ and $\mathcal{Q}_{V A}^{ \pm[2]}$. We first notice that the $S U(4)$ flavour symmetry of the massless theory prohibits mixing between + and - operators. Absence of mixing with operators having tensor structures other than $V A+A V$ is proved by identifying the various symmetries that forbid them. The result is

- $\mathcal{P}_{5}$ forbids $V V \pm A A, S S \pm P P$ and $T T$.

- $\operatorname{Ex}(d, s) \times \mathcal{C}$ forbids $S P+P S$ and $T \tilde{T}$.

- $\operatorname{Ex}(d, s) \times \mathcal{C} \times S U(4)_{v}$ forbids $S P-P S$ and $V A-A V$.

The analysis of the mixing properties of $\mathcal{Q}_{V A}^{ \pm[1]}$ $\left(\mathcal{Q}_{V A}^{ \pm[3]}\right)$ is immediately brought back to the case of $\mathcal{Q}_{V A}^{ \pm[0]}\left(\mathcal{Q}_{V A}^{ \pm[2]}\right)$ discussed before, by the observation that the change of variables induced by $\mathcal{R}_{5}$ on the valence quarks $u^{[1]}$ and $c^{[1]}\left(u^{[3]}\right.$ and $\left.c^{[3]}\right)$ changes sign to $r_{u^{[1]}}$ and $r_{c^{[1]}}\left(r_{u^{[3]}}\right.$ and $\left.r_{c^{[3]}}\right)$, making them equal to $r_{u^{[0]}}$ and $r_{c^{[0]}}\left(r_{u^{[2]}}\right.$ and $\left.r_{c^{[2]}}\right)$.

\subsection{3. $K \rightarrow \pi$ with no mixing}

By the use of soft-pion theorems one can relate $K \rightarrow \pi \pi$ to $K \rightarrow \pi$ amplitudes [399] in the chiral limit with the advantage that only three-point correlators need be evaluated and no problems with final state interactions occur.

In this approach the relevant target matrix elements are, for instance, $\left\langle\pi^{+}, \mathbf{q}\left|\mathcal{O}_{V V+A A}^{ \pm}\right| K^{+}, \mathbf{p}\right\rangle$. They can be extracted from the correlators

$C_{ \pm, K \pi}^{\mathrm{QCD} 4}=\left.\left\langle\Phi_{\pi}(x) \mathcal{O}_{V V+A A}^{ \pm}(0) \Phi_{K}^{\dagger}(y)\right\rangle\right|^{\mathrm{QCD} 4}$,

$2 \mathcal{O}_{V V+A A}^{ \pm}=\left[\left(\bar{s} \gamma_{\mu} u\right)\left(\bar{u} \gamma_{\mu} d\right) \pm\left(\bar{s} \gamma_{\mu} d\right)\left(\bar{u} \gamma_{\mu} u\right)+(36)\right.$ $\left.+\left(\bar{s} \gamma_{\mu} \gamma_{5} u\right)\left(\bar{u} \gamma_{\mu} \gamma_{5} d\right) \pm\left(\bar{s} \gamma_{\mu} \gamma_{5} d\right)\left(\bar{u} \gamma_{\mu} \gamma_{5} u\right)\right]-[u \leftrightarrow c]$.

Noting that, if QCD4 and $4 s 10 v$ are regularized 
in the same way, the Wick theorem ensures the equality of all contractions, we conclude that the same information is contained in

$C_{ \pm, K \pi}^{4 s 10 v}=\left.\left\langle\Phi_{\pi}(x) \mathcal{Q}_{V V+A A}^{ \pm}(0) \Phi_{K}^{\dagger}(y)\right\rangle\right|^{4 s 10 v}$,

As before, there is a suitable regularization of $4 s 10 v$ which makes the operators $\mathcal{Q}_{V V+A A}^{ \pm}$multiplicatively renormalizable. Besides taking all the renormalized quark masses of the same flavour equal, one needs to choose the Wilson parameters of the OS valence quarks obeying the relation

$$
\begin{aligned}
& r_{d}=-r_{s}=r_{u^{[0]}}=-r_{u^{[1]}}=r_{u^{[2]}}=-r_{u^{[3]}}= \\
& =r_{c^{[0]}}=-r_{c^{[1]}}=r_{c^{[2]}}=-r_{c^{[3]}} .
\end{aligned}
$$

We notice that the only difference with respect to the choice (34) is the sign change in front of $r_{s}$. We will call this regularization $4 s 10 v^{L \star}$.

The proof of this statement follows from the invariance of expectation values under the change of fermionic integration variables induced by the transformation $s \rightarrow s^{\prime}=\gamma_{5} s, \bar{s} \rightarrow \bar{s}^{\prime}=-\bar{s} \gamma_{5}$ on the valence strange quark (the associated ghost fields should be simultaneously transformed in the appropriate way [5]). Under this change of variables the action of $4 s 10 v^{L \star}$ goes over to the action of the $4 s 10 v^{L}$ theory of sect. 4.2.2 modulo the change of sign of the valence quark mass, $m_{s}$. At the same time $\mathcal{Q}_{V V+A A}^{ \pm} \rightarrow \mathcal{Q}_{V A}^{ \pm}$ and $\left(m_{s}+m_{d}\right) \bar{s} d \rightarrow\left(-m_{s}+m_{d}\right) \bar{s} \gamma_{5} d$. We are thus exactly in the situation discussed in sect. 4.2.2 except that now the operators $[\bar{s} d$, $\bar{s} \sigma \cdot F d]$ will appear multiplied by the mass factors $\left(m_{c}^{2}-m_{u}^{2}\right)\left(m_{s}+m_{d}\right)$ and the operators $\left[\bar{s} \gamma_{5} d\right.$, $\left.\bar{s} \gamma_{5} \sigma \cdot F d\right]$ by $\left(m_{c}^{2}-m_{u}^{2}\right)\left(m_{s}^{2}-m_{d}^{2}\right)$.

This argument also proves that the operators $\mathcal{O}_{V V+A A}^{ \pm}$in QCD4 and $\mathcal{Q}_{V V+A A}^{ \pm}$in $4 s 10 v^{L *}$ (can be taken to) have equal renormalization constant and the same as $\mathcal{Q}_{V A}^{ \pm}$in $4 s 10 v^{L}$.

We conclude by stressing that in all the applications considered in sect. 团 full $\mathrm{O}\left(a^{2 k+1}\right), k \geq 0$, improvement is ensured by the symmetry of the regularized actions under $\mathcal{P} \times \mathcal{D}_{d} \times(M \rightarrow-M)$.

\section{Chirally enhanced cutoff effects}

Although, as we have seen, in Mtm-LQCD odd power discretization effects are absent or easily eliminated 234.5, it turns out that correlators are affected by dangerous artifacts of relative order $a^{2 k}, k \geq 1$, which are enhanced by inverse powers of the (squared) pion mass, as the latter becomes small 6. In fact, when analyzed in terms of the Symanzik expansion, lattice expectation values exhibit, as $m_{\pi}^{2} \rightarrow 0$, what we will call "infrared (IR) divergent" cutoff effects with a behaviour of the form ( $2 k \geq h \geq 1, k, h$ integers)

$\left.\langle O\rangle\right|_{m_{q}} ^{L}=\left.\langle O\rangle\right|_{m_{q}} ^{\text {cont }}\left[1+\mathrm{O}\left(\frac{a^{2 k}}{\left(m_{\pi}^{2}\right)^{h}}\right)\right]$,

where we have assumed that the lattice correlator admits a non-trivial continuum limit. Powers of $\Lambda_{\mathrm{QCD}}$ required to match physical dimensions are often understood in this section.

We want to prove that artifacts of the type (39) are reduced to terms that are at worst of order $a^{2}\left(a^{2} / m_{\pi}^{2}\right)^{k-1}, k \geq 1$, if the action is $\mathrm{O}(a)$ improved $a$ la Symanzik or, alternatively, if the critical mass is chosen in some "optimal" way.

The idea that a suitable definition of critical mass exists which can lead to a smoothing out of chirally enhanced lattice artifacts or perhaps be of help in getting improvement was already put forward in the context of lattice $\chi \mathrm{PT}$ in refs. 10. and 11, respectively.

An important consequence of this analysis is that the strong (order of magnitude) inequality $m_{q}>a \Lambda_{\mathrm{QCD}}^{2}$, invoked in ref. [3], can be relaxed to the weaker relation $m_{q}>a^{2} \Lambda_{\mathrm{QCD}}^{3}$, before large cutoff effects are possibly met while lowering the quark mass at fixed $a$. The works of refs. 1011, and most recently refs. [1213, all based on lattice $\chi \mathrm{PT}$, lead to essentially equivalent conclusions about cutoff effects in pion quantities in the parameter region $m_{q}>a^{2} \Lambda_{\mathrm{QCD}}^{3}$. They also yield interesting predictions on the possible Wilson fermion phase scenarios [1819 and observables, when $m_{q}$ is of order $a^{2}$ or smaller.

A nice numerical demonstration of the effectiveness of Mtm-LQCD in killing $\mathrm{O}(a)$ discretization errors and the ability of the optimal choice of the critical mass in diminishing the magnitude of lattice artifacts at small quark mass can be found in refs. 424323 . As for Mtm-LQCD with cloverimproved quark action, the promising quenched tests presented some years ago in 44 have been recently extended in 45 down to pion masses of 
$300 \mathrm{MeV}$ or lower, confirming the absence of large cutoff effects.

\subsection{Symanzik analysis of "IR divergent" cutoff artifacts}

The study of discretization artifacts affecting lattice correlators in Mtm-LQCD can be elegantly made in the language of the Symanzik expansion. We focus here on Mtm-LQCD with two mass degenerate flavours. Its fermionic action is given by eq. (1) with $\omega=\pi / 2$. A full analysis of cutoff effects beyond $\mathrm{O}(a)$ is extremely complicated. Fortunately it is not necessary, if we limit the discussion to the terms that are enhanced as the quark mass $m_{q}$ is decreased.

- The Symanzik LEEA of Mtm-LQCD - The low energy effective action (LEEA), $S_{\text {Sym }}$, of the theory can be written in the form

$S_{\mathrm{Sym}}=\int d^{4} y\left[\mathcal{L}_{4}(y)+\sum_{j=0}^{\infty} a^{j} \ell_{4+j}(y)\right]$,

where $\mathcal{L}_{4}=\frac{1}{2 g_{0}^{2}} \operatorname{tr}(F \cdot F)+\bar{\psi}\left(\gamma \cdot D+m_{q}\right) \psi$ is the target continuum 2-flavour QCD Lagrangian. Based on the symmetries of Mtm-LQCD a number of interesting properties enjoyed by $S_{\text {Sym }}$ can be proved which are summarized below.

1. Lagrangian densities of even dimension, $\ell_{4+2 k}$, in eq. (40) are parity-even, while terms of odd dimension, $\ell_{5+2 k}$, are parity-odd and twisted in iso-spin space. Thus the latter have the quantum numbers of the neutral pion.

2. The term of order $a$ in eq. (45), $\ell_{5}$, is given (on-shell) by the linear combination

$\ell_{5}=\delta_{5, S W} \ell_{5, S W}+\delta_{5, m^{2}} \ell_{5, m^{2}}+\delta_{5, e} \ell_{5, e}$,

$\ell_{5, S W}=\frac{i}{4} \bar{\psi}[\sigma \cdot F] i \gamma_{5} \tau_{3} \psi, \quad \ell_{5, m^{2}}=m_{q}^{2} \bar{\psi} i \gamma_{5} \tau_{3} \psi$,

$\ell_{5, e}=\Lambda_{\mathrm{QCD}}^{2} \bar{\psi} i \gamma_{5} \tau_{3} \psi$,

where $\delta_{5, S W}, \delta_{5, m^{2}}$ and $\delta_{5, e}$ are dimensionless coefficients, odd in $r$. The operator $\ell_{5, e}$ arises from the need to describe order $a$ uncertainties entering any non-perturbative determination of the critical mass and goes together with $\ell_{5, S W}$. Both $\ell_{5, S W}$ and $\ell_{5, e}$ could be made to disappear from (40) by introducing in the Mtm-LQCD action the SW (clover)-term [14] with the appropriate non-perturbatively determined $c_{S W}$ coeffi- cient [15] and at the same time setting the critical mass to its correspondingly $\mathrm{O}(a)$ improved value.

3. Higher order ambiguities in the critical mass, which will all contribute to $\mathcal{L}_{\text {odd }}$, are described by terms proportional to odd powers of $a$ of the kind

$a^{2 k+1} \delta_{5+2 k, e} \ell_{5+2 k, e}=$
$=a^{2 k+1} \delta_{5+2 k, e}\left(\Lambda_{\mathrm{QCD}}\right)^{2 k+2} \bar{\psi} i \gamma_{5} \tau_{3} \psi, k \geq 1$.

- Describing lattice correlators beyond $O(a)$ We are interested in the Symanzik description of the lattice artifacts affecting the correlator $\left.\langle O\rangle\right|_{m_{q}} ^{L}$, where $O$ has continuum vacuum quantum numbers so as to yield a non trivially vanishing result as $a \rightarrow 0$. Schematically we write

$$
\begin{aligned}
& \left.\langle O(x)\rangle\right|_{m_{q}} ^{L}=\left\langle\left[O(x)+\Delta_{\text {odd }} O(x)+\Delta_{\text {even }} O(x)\right]\right. \\
& \left.e^{-\int d^{4} y\left[\mathcal{L}_{\text {odd }}(y)+\mathcal{L}_{\text {even }}(y)\right]}\right\rangle\left.\right|_{m_{q}} ^{\text {cont }}, \\
& \mathcal{L}_{\text {odd }}=\sum_{k=0}^{\infty} a^{2 k+1} \ell_{5+2 k}, \mathcal{L}_{\text {even }}=\sum_{k=1}^{\infty} a^{2 k} \ell_{4+2 k} .
\end{aligned}
$$

The operators $\Delta_{\text {odd }} O\left(\Delta_{\text {even }} O\right)$ have an expansion in odd (even) powers of $a$. They can be viewed as the operators necessary for the improvement of the matrix elements of $O$ [2815]. To ensure automatic improvement $[3$ we shall assume that $O$ is parity invariant in which case its Symanzik expansion will contain only even powers of $a$.

- Pion poles and "IR divergent" cutoff effects Although a complete analysis of all the "IR divergent" cutoff effects is very complicated, the structure of the leading ones $(h=2 k$ in eq. (39) $)$ is rather simple, as they only come from continuum correlators where $2 k$ factors $\int d^{4} y \mathcal{L}_{\text {odd }}(y)$ are inserted. More precisely the leading "IR divergent" cutoff effects are identified on the basis of the following result 6 .

In the Symanzik expansion of $\left.\langle O\rangle\right|_{m_{q}} ^{L}$ at or$\operatorname{der} a^{2 k}(k \geq 1)$ there appear terms with a $2 k$-fold pion pole and residues proportional to $\left|\left\langle\Omega\left|\mathcal{L}_{\text {odd }}\right| \pi^{0}(\mathbf{0})\right\rangle\right|^{2 k}$, where $\langle\Omega|$ and $\left|\pi^{0}(\mathbf{0})\right\rangle$ denote the vacuum and the one- $\pi^{0}$ state at zero threemomentum, respectively. Putting different factors together, each one of these terms can be seen 
to be schematically of the form $\left(\mathcal{L}_{\text {odd }}=\mathrm{O}(a)\right)$

$\left[\left(\frac{1}{m_{\pi}^{2}}\right)^{2 k}\left(\xi_{\pi}\right)^{2 k} \mathcal{M}\left[O ;\left\{\pi^{0}(\mathbf{0})\right\}_{2 k}\right]\right]_{m_{q}}^{\text {cont }}$,

$\xi_{\pi}=\left|\left\langle\Omega\left|\mathcal{L}_{\text {odd }}\right| \pi^{0}(\mathbf{0})\right\rangle\right|_{m_{q}}^{\text {cont }}$,

where we have generically denoted by $\mathcal{M}\left[O ;\left\{\pi^{0}(\mathbf{0})\right\}_{2 k}\right]$ the $2 k$-particle matrix elements of $O$, with each external leg being a zero threemomentum neutral pion.

Less "IR divergent" cutoff effects (those with $h$ strictly smaller than $2 k$ in (39) ) come either from terms with some extra $\int d^{4} y \mathcal{L}_{\text {even }}(y)$ insertions or from contributions of more complicated intermediate states other than straight zero threemomentum pions or from both. In all these cases one gets extra $a^{2}$ powers, not all "accompanied" by corresponding $1 /\left(m_{\pi}^{2}\right)^{2}$ factors.

It is important to stress that the appearance of pion poles like the ones in (46) in no way means that the lattice correlators diverge as $m_{q} \rightarrow 0$, but only that the Symanzik expansion we have employed appears to have a finite radius of convergence (on this point see the remarks in [13]).

\subsection{Reducing "IR divergent" cutoff effects}

Recalling that $\mathcal{L}_{\text {odd }}=a \ell_{5}+\mathrm{O}\left(a^{3}\right)$, the previous analysis shows that at leading order in $a$ the residue of the most severe multiple pion poles is proportional to $\left|\left\langle\Omega\left|\ell_{5}\right| \pi^{0}(\mathbf{0})\right\rangle\right|^{2 k}$. It is an immediate conclusion then that the leading "IR divergent" cutoff effects can all be eliminated from lattice data if we can either reduce $\ell_{5}$ to only the $\ell_{5, m^{2}}$ term in (41) or set $\xi_{\pi}$ to zero.

- Improving the Mtm-LQCD action - The obvious, field-theoretical way to achieve the first goal consists in adding the clover term 141528 to the Mtm-LQCD action. In this case lattice correlators will admit a Symanzik description in terms of a LEEA where the operators $\ell_{5, S W}$ and $\ell_{5, e}$ are absent, and in $\ell_{5}$ only $\ell_{5, m^{2}}$ survives. The left-over contributions arising from the insertions of $\ell_{5, m^{2}}$ yield terms that are at most of order $\left(a m_{q}^{2} / m_{\pi}^{2}\right)^{2 k} \simeq\left(a m_{q}\right)^{2 k}$, hence negligible in the chiral limit. It is instead the next odd operator in the expansion (401), $a^{3} \ell_{7}$, which comes into play.

A combinatoric analysis based on the structure of the non-leading "IR divergent" cutoff effects reveals that the worst lattice artifacts left behind in correlators after the "clover cure" are of the kind $a^{2}\left(a^{2} / m_{\pi}^{2}\right)^{k-1}, k \geq 1$.

- Optimal choice of the critical mass - The alternative strategy to kill the leading "IR divergent" cutoff effects consists in leaving the MtmLQCD action unimproved, but fixing the critical mass through the condition

$$
\lim _{m_{q} \rightarrow 0} \xi_{\pi}\left(m_{q}\right)=\lim _{m_{q} \rightarrow 0}\left|\left\langle\Omega\left|\mathcal{L}_{\text {odd }}\right| \pi^{0}(\mathbf{0})\right\rangle\right|_{m_{q}}^{\text {cont }}=0 .
$$

The meaning of (48) is simple. It amounts to fix, for $k \geq 0$, the order $a^{2 k+1}$ contribution in the counter-term, $M_{\mathrm{cr}} \bar{\psi}^{L} i \gamma_{5} \tau_{3} \psi^{L}$, so that its vacuum to one- $\pi^{0}(\mathbf{0})$ matrix element compensates, in the limit $m_{q} \rightarrow 0$, the similar matrix element of the sum of all the other operators making up $\ell_{5+2 k}$.

Concrete procedures designed to implement condition (48) in practice were discussed in 6]. They are all based on the idea of determining the critical mass by requiring the lattice correlator $\left.a^{3} \sum_{\mathbf{x}}\left\langle V_{0}^{2}(x) P^{1}(0)\right\rangle\right|_{m_{q}} ^{L}\left(x_{0} \neq 0\right)$ to vanish in the chiral limit $\left(V_{0}^{2}=\bar{\psi} \gamma_{0} \frac{\tau_{2}}{2} \psi\right.$ is the vector current with iso-spin index 2 and $P^{1}=\bar{\psi} \gamma_{5} \frac{\tau_{1}}{2} \psi$ the pseudo-scalar density with iso-spin index 1 ). In the continuum this correlator is zero by parity for any value of $m_{q}$. On the lattice the breaking of parity (and iso-spin) due to the twisting of the Wilson term makes it non-vanishing by $\mathrm{O}(a)$ discretization artifacts, which have the form of a power series expansion in $\xi_{\pi}$.

The important conclusion of the analysis presented in $[6$ is that it is not necessary (nor possible) to really go to $m_{q} \rightarrow 0$. It is enough to have the critical mass determined by the vanishing of the previous correlator at the smallest available $m_{q}$-value, say $m_{q}^{\min }$, provided it fulfills the order of magnitude inequality $m_{q}^{\min }>a^{2}$. Under this condition, for any $m_{q} \geq m_{q}^{\min }$ we will have $\xi_{\pi}\left(m_{q}\right)=\mathrm{O}\left(a m_{\pi}^{2}\right)$. Substituting this estimate into the leading "IR divergent" terms, we see that they are reduced to finite $\mathrm{O}\left(a^{2 k}\right)$ terms. As for the sub-leading terms, a non-trivial diagrammatic analysis shows that the worst of them, left behind after the "optimal critical mass cure", are reduced to only $a^{2}\left(a^{2} / m_{\pi}^{2}\right)^{k-1}, k \geq 1$, effects, just like in the case where the clover term is employed.

The results reached in this section show that the continuum extrapolation of lattice data 
should be smooth at least down to values of the quark mass satisfying the order of magnitude inequality $m_{q}>a^{2} \Lambda_{\mathrm{QCD}}^{3}$, if the clover term is added to the lattice action, as well as if the critical mass is adjusted to its "optimal" value.

\subsection{The lattice GMOR relation}

A workable way to numerically estimate the minimal value of the quark mass that can be safely simulated at fixed $a$ can be obtained by considering the behaviour of the charged pion mass as a function of $m_{q}$. It turns out, in fact, that in Mtm-LQCD there are Ward-Takahashi identities (WTI's) which take exactly the form they have in the formal continuum theory. From them a lattice GMOR relation can be derived.

To see how this works we recall that in MtmLQCD the 1-point split axial currents, $\hat{A}_{\mu}^{b}$, with iso-spin index $b=1,2$ are exactly conserved at $m_{q}=023$. This implies the validity of the WTI's $\left(\tau^{ \pm}=\left(\tau_{1} \pm i \tau_{2}\right) / 2\right)$

$\left.\left\langle\left[\partial_{\mu}^{*} \hat{A}_{\mu}^{ \pm}-2 m_{q} P^{ \pm}\right](x) P^{\mp}(0)\right\rangle\right|_{m_{q}} ^{L}=\left.\left\langle S^{0}(0)\right\rangle\right|_{m_{q}} ^{L} \delta_{x, 0}(49)$

$\hat{A}_{\mu}^{ \pm}=\hat{A}_{\mu}^{1} \pm i \hat{A}_{\mu}^{2}, P^{ \pm}=\bar{\psi}^{L} \gamma_{5} \tau^{ \pm} \psi^{L}, S^{0}=\bar{\psi}^{L} \psi^{L} .(50)$

After space-time integration, one gets $\left(m_{q} \neq 0\right)$

$\left.2 m_{q} a^{4} \sum_{x}\left\langle P^{ \pm}(x) P^{\mp}(0)\right\rangle\right|_{m_{q}} ^{L}=-\left.\left\langle S^{0}(0)\right\rangle\right|_{m_{q}} ^{L}$.

Although, as the WTI (51) itself shows, there is no mixing between $S^{0}$ and the identity operator with a cubically (or a linearly) divergent coefficient, there is still room for a quadratically divergent term proportional to $m_{q}$. Indeed, the l.h.s. of (51) can be written as the sum of a piece where intermediate states are inserted plus a divergent contribution of the kind $m_{q} / a^{2}$ coming from the (integrated) short-distance singularity of $\left\langle P^{ \pm}(x) P^{\mp}(0)\right\rangle$ at $x=0$. This term should be brought to the r.h.s., thus leading to the subtracted expression of the chiral condensate.

We can now repeat on the lattice the argument that in the continuum leads to the classical GMOR relation. We will work under the assumption that spontaneous chiral symmetry breaking occurs in the limiting continuum theory, i.e. under the assumption that

$\Sigma \equiv-\left.\left\langle\Omega\left|S^{0}\right| \Omega\right\rangle\right|_{m_{q}=0} ^{\text {cont }} \neq 0$.
After inserting a complete set of states in the l.h.s. of eq. (51), we get

$\left.m_{\pi^{ \pm}}^{2}\right|_{m_{q}} ^{L}=\left.2 m_{q} \frac{\left|\left\langle\Omega\left|P^{ \pm}\right| \pi^{ \pm}\right\rangle\right|^{2}}{\left[-\left\langle\Omega\left|S_{\mathrm{sub}}^{0}\right| \Omega\right\rangle\right]}\right|_{m_{q}} ^{L}+\ldots$,

where we have explicitely written only the contribution coming from the pion pole. Dots are terms due to the intermediate states that stay massive as $m_{q} \rightarrow 0$, as well as terms vanishing with $m_{q}$ faster than linearly. The subscript "sub" is to remind us that it is the properly subtracted chiral condensate that enters this equation. Notice that, as expected, the the r.h.s. of eq. (53) is a finite, renormalized, quantity in the limit $a \rightarrow 0$.

Once the leading "IR divergent" cutoff effects have been canceled out, the use of the Symanzik expansion in the r.h.s. of eq. (53) yields

$\left.m_{\pi \pm}^{2}\right|_{m_{q}} ^{L}=2 m_{q} \frac{G_{\pi}^{2}}{\Sigma}\left[1+a^{2} \sum_{\ell>0} b_{\ell}\left(\frac{a^{2}}{m_{q}}\right)^{\ell}\right]^{\mathrm{cont}}+\ldots$

where $G_{\pi}=\left|\left\langle\Omega\left|P^{ \pm}\right| \pi^{ \pm}\right\rangle\right|_{m_{q}=0}^{\text {cont }}$. In eq. (54) dots denote less dangerous "IR divergent" lattice artifacts compared to those explicitely shown, as well as contributions of higher order in $m_{q}$. In getting eq. (54) we have used the fact that the continuum limit of $-\left\langle\Omega\left|S_{\text {sub }}^{0}\right| \Omega\right\rangle^{L}$ at vanishing quark mass is $\Sigma \neq 0$ (see eq. (52)).

From the above analysis it follows that, in the region where the series in eq. (54) converges (i.e. at least down to masses where the order of magnitude inequality $m_{\pi}^{2} \sim m_{q}>a^{2}$ is still satisfied), the squared mass of the charged lattice pion is linear in $m_{q}$ (up to very small deviations). Thus, vice-versa, we can imagine to use deviations from the established linear behaviour that are possibly seen at small $m_{q}$ as a workable criterion to determine the minimal value of $m_{q}$ at which simulations can be performed before being set-off by discretization effects.

5.4. Artifacts on hadronic energies and $f_{\pi}$

We wish to conclude by discussing some peculiar features concerning the magnitude of the $\mathrm{O}\left(a^{2}\right)$ cutoff effects on hadron energies (in particular masses) and on the pion decay constant. 


\subsubsection{Hadron energies}

In the language of the Symanzik expansion discretization artifacts on hadronic energies are described by a set of diagrams where at least one among the inserted $\int \mathcal{L}_{\text {odd }}$ factors gets absorbed in a multi-particle irreducible matrix element, with the consequence that it is not available for producing a pion pole. As a consequence, at fixed order in $a$, the most "IR divergent" lattice corrections to continuum hadronic energies contain one overall $1 / m_{\pi}^{2}$ factor less than the leading "IR divergent" cutoff effects generically affecting correlators. For instance, at order $a^{2}$ the difference between lattice and continuum energy of the hadron $\alpha_{n}$ reads [6] (the label $\mathbf{q}$ specifying the three-momentum of the state $\left|\alpha_{n}(\mathbf{q})\right\rangle$ is omitted)

$\left.\Delta E_{\alpha_{n}}(\mathbf{q})\right|_{a^{2}} \propto$

$\frac{a^{2}}{m_{\pi}^{2}} \operatorname{Re} \frac{\left\langle\Omega\left|\ell_{5}\right| \pi^{0}(\mathbf{0})\right\rangle\left\langle\pi^{0}(\mathbf{0}) \alpha_{n}\left|\ell_{5}\right| \alpha_{n}\right\rangle}{2 E_{\alpha_{n}}(\mathbf{q})}+\left.\mathrm{O}\left(a^{2}\right)\right|_{m_{q}} ^{\mathrm{cont}}$,

where $\mathrm{O}\left(a^{2}\right)$ denotes "IR finite" corrections. It should be noted that $\left.\Delta E_{\alpha_{n}}(\mathbf{q})\right|_{a^{2}}$ is reduced to a plain $\mathrm{O}\left(a^{2}\right)$ "IR finite" correction after anyone of the two "cures" described in Sect. 5.2

Specializing (55) to the case of pions, one obtains the interesting result that the difference between charged and neutral pion (square) masses is a finite $\mathrm{O}\left(a^{2}\right)$ quantity even if the critical mass has not been set to its optimal value or the clover term has not been introduced. The reason is that the leading "IR divergent" contributions shown in (55) are equal for all pions (as one can prove by standard soft pion theorems [46]), hence cancel in the (square) mass difference. This conclusion is in agreement with detailed results from chiral perturbation theory (see refs. 20] and [10]), as well as with the first numerical estimates of the pion square mass splitting in Mtm-LQCD 22.

\subsubsection{Pion decay constant}

Data on a (quenched) computation of $f_{\pi}$, carried out in Mtm-LQCD by using the value of the critical mass obtained from the vanishing of the pion mass, show cutoff effects which tend to increase as the quark mass is lowered [4]. In particular data lie lower than the straight line extrapolation drawn from larger masses. This behaviour (called "bending phenomenon" in ref. 47]) sets in at values of the bare quark mass around $a \Lambda_{\mathrm{QCD}}^{2}$, $\Lambda_{\mathrm{QCD}} \sim 200 \mathrm{MeV}$. A recent scaling test of MtmLQCD [43] furthermore shows that the "bending phenomenon" is an $\mathrm{O}\left(a^{2}\right)$ cutoff effect with a magnitude which increases as $m_{\pi^{ \pm}}^{2}$ is lowered.

In refs. 474243 the lattice pion decay constant, $f_{\pi}^{L}$, was determined using the formula 23 .

$f_{\pi}^{L}\left(m_{\pi^{ \pm}}^{2}\right)=\left.2 m_{q} \frac{\left\langle\Omega\left|P^{ \pm}\right| \pi^{\mp}\right\rangle}{m_{\pi^{ \pm}}^{2}}\right|_{m_{q}} ^{L}$,

from which $f_{\pi}^{L}$ is seen to be the ratio of two lattice quantities. It should be noted that the matrix element in the numerator of eq. (56) is affected by leading "IR divergent" lattice artifacts which are softened by an extra $m_{\pi}^{2} \sim m_{q}$ factor. This property can be traced back to the invariance of the correlator $\left.\left\langle P^{ \pm}(x) P^{\mp}(y)\right\rangle\right|_{m_{q}} ^{L}$ under axial rotations around the third iso-spin direction. One can, in fact, always think of having chosen the $\mathrm{O}(a)$ - rotation angle such to bring the critical mass to its optimal value, at the price of shifting the bare quark mass by a term of order $a^{2} / m_{q}$. Also $\left(m_{\pi^{ \pm}}^{L}\right)^{2}$ is affected by leading "IR divergent" cutoff effects softened by an extra $m_{\pi}^{2}$ factor. The latter, however, drops out in the ratio $\left(m_{\pi^{ \pm}}^{L}\right)^{2} / m_{q}$ entering eq. (56).

With anyone of the two cures described in sect. 5.2 lattice artifacts are reduced to terms that are only of the kind $a^{2} \sum_{\ell \geq 0} c_{\ell}\left(a^{2} / m_{q}\right)^{\ell}[\underline{6}$.

Indeed, a beautiful confirmation of the validity of the analysis of "IR divergent" cutoff effects presented here comes from the fact that, when the critical mass is set at its optimal value, no bending effect is anymore visible in the $f_{\pi}^{L}$ data obtained in ref. 4243] (see also [23]).

\section{Conclusions and outlook}

In this lecture we have reviewed the main properties of Mtm-LQCD with the purpose of underlining the features that makes it an appealing regularization for realistic simulations of QCD4 at adequately small pion masses. Actually explorative unquenched simulations of QCD4 with renormalized masses satisfying $m_{u}=m_{d}<m_{s}<$ $m_{c}$ have already started [48].

We have not discussed the very important is- 
sue of meta-stabilities that on too coarse lattices appear to affect unquenched simulations and prevent reaching sufficiently light pions 2148, at least in the Singleton-Sharpe scenario [19].

In the language of lattice $\chi \mathrm{PT}$ the strength of meta-stabilities and the magnitude of the attainable minimal pion mass are features that are both controlled by the magnitude of the coefficient (called $c_{2}$ in refs. 1920) which in the chiral Lagrangian multiplies the term $\left(\operatorname{Tr}\left[\Sigma+\Sigma^{\dagger}\right]\right)^{2}$ describing $\mathrm{O}\left(a^{2}\right)$ lattice artifacts in correlators. Ideally, one thus would like to have $c_{2}=0$ as a function of $g_{0}^{2}$. This situation is not peculiar of Mtm-LQCD. Similar features arise for generic values of the twisting angle and even for plain (clover) Wilson fermions a non-vanishing $c_{2}$ might turn out to be problematic for simulations at fixed $a$ if too small pion masses are taken [19].

Comparing $\chi \mathrm{PT}$ analysis with results from the Symanzik LEEA approach, one can prove that $c_{2}$ is proportional to the matrix element $a^{2}\left\langle\pi^{0}(\mathbf{0})\left|\ell_{6}^{\chi-\mathrm{br}}\right| \pi^{0}(\mathbf{0})\right\rangle$, where $\ell_{6}^{\chi-\mathrm{br}}$ is the chirally breaking piece of $\ell_{6}$ (see eq. (45)). This observation suggests that a possible way to enforce the condition $c_{2}=0$ is to modify the gauge action by adding to the standard plaquette term further dimension 6 operators. For instance, one can imagine adding $b\left(g_{0}^{2}\right) P_{2 \times 1}$, where $b\left(g_{0}^{2}\right)$ is an adjustable coefficient and $P_{2 \times 1}$ the minimal rectangular plaquette. This term, through the mixing induced by the chirally breaking twisted Wilson term, will modify, among others, all dimension 6 operators of the Symanzik LEEA, including those that break chiral invariance. Since $c_{2}$ also controls the square mass difference between charged and neutral pion [201011], $\Delta m_{\pi}^{2}=m_{\pi^{ \pm}}^{2}-m_{\pi^{0}}^{2} \propto c_{2}$, the idea to ease Mtm-LQCD simulations is then to fix $b\left(g_{0}^{2}\right)$ as a function of $g_{0}^{2}$ by imposing the condition $\Delta m_{\pi}^{2}=0$.

Acknowledgments - G.C.R. thanks the Organizers for the exciting atmosphere of the workshop and the wonderful hospitality in Cyprus.

\section{REFERENCES}

1. P.H. Ginsparg and K.G. Wilson, Phys. Rev. D25 (1982) 2649.
2. R. Frezzotti, P.A. Grassi, S. Sint and P. Weisz, Nucl. Phys. B (Proc. Suppl.) 83 (2000) 941 and JHEP 0108 (2001) 058;

R. Frezzotti, S. Sint and P. Weisz, JHEP 0107 (2001) 048;

M. Della Morte, R. Frezzotti, J. Heitger and S. Sint, JHEP 0110 (2001) 041;

R. Frezzotti and S. Sint, Nucl. Phys. B (Proc. Suppl.) 106 (2002) 814;

R. Frezzotti, Nucl. Phys. B (Proc. Suppl.) 119 (2003) 140 and 140 (2005) 134.

3. R. Frezzotti and G.C. Rossi, JHEP 0408 (2004) 007 and Nucl. Phys. B (Proc. Suppl.) 129 (2004) 880.

4. R. Frezzotti and G.C. Rossi, Nucl. Phys. B (Proc. Suppl.) 128 (2004) 193.

5. R. Frezzotti and G.C. Rossi, JHEP 0410 (2004) 070 and hep-lat/0509155.

6. R. Frezzotti, G. Martinelli, M. Papinutto and G.C. Rossi, hep-lat/0503034

7. F. Farchioni, K. Jansen, I. Montvay, E. Scholz, L. Scorzato, A. Shindler, N. Ukita, C. Urbach and I. Wetzorke, Eur. Phys. J. C42 (2005) 73 .

8. K. Österwalder and E. Seiler, Ann. of Phys. 110 (1978) 440.

9. M. Bochicchio, L. Maiani, G. Martinelli, G.C. Rossi and M. Testa, Nucl. Phys. B262 (1985) 331 .

10. S.R. Sharpe and J.M.S. Wu, Phys. Rev. D71 (2005) 074501 and Nucl. Phys. B (Proc. Suppl.) 140 (2005) 323.

11. S. Aoki and O. Bär, Phys. Rev. D70 (2004) 116011 .

12. S. Aoki and O. Bär, hep-lat/0509002

13. S.R. Sharpe, hep-lat/0509009.

14. B. Sheikholeslami and R. Wohlert, Nucl. Phys. B259 (1985) 572.

15. M. Lüscher, S. Sint, R. Sommer and P. Weisz, Nucl. Phys. B478 (1996) 365;

M. Lüscher, S. Sint, R. Sommer, P. Weisz and U. Wolff, Nucl. Phys. B491 (1997) 323.

16. R. Frezzotti and G.C. Rossi, hep-lat/0507030

17. S. Sint, Frascati workshop (2005).

18. S. Aoki, Phys. Rev. D30 (1984) 2653 and Phys. Rev. Lett. 57 (1986) 3136.

19. S.R. Sharpe and R. Singleton, Jr., Phys. Rev. D58 (1998) 074501. 
20. S.R. Sharpe and J.M.S. Wu, Phys. Rev. D70 (2004) 094029;

G. Münster, JHEP 0409 (2004) 035;

L. Scorzato, Eur. Phys. J. C37 (2004) 445.

21. F. Farchioni, R. Frezzotti, K. Jansen, I. Montvay, G.C. Rossi, E. Scholz, A. Shindler, N. Ukita, C. Urbach, I. Werzorke, Eur. Phys. J. C39 (2005) 421.

22. K. Jansen et al. [ $\chi \mathrm{LF}$ Collaboration], hep-lat/0507032 and hep-lat/0509036.

23. A. Shindler, hep-lat/0511002

24. K. Symanzik, in "New Developments in Gauge Theories", page 313, Eds. G. 't Hooft et al., Plenum (New York, 1980); "Some topics in quantum field theory" in "Mathematical Problems in Theoretical Physics", Eds. R. Schrader et al., Lectures Notes in Physics, Vol. 153, Springer (New York, 1982) and Nucl. Phys. B226 (1983) 187 and 205.

25. M. Della Morte and M. Luz, hep-lat/0510092 K. Nagai and K. Jansen, hep-lat/0510076

26. A. Donini, V. Giménez, G. Martinelli, M. Talevi and A. Vladikas, Eur. Phys. J. C10 (1999) 121.

27. G. Martinelli, G.C. Rossi, C.T. Sachrajda, S.R. Sharpe, M. Talevi and M. Testa, Phys. Lett. B411 (1997) 141 and Nucl. Phys. B611 (2001) 3

28. G. Heatlie, G. Martinelli, C. Pittori, G.C. Rossi and C.T. Sachrajda, Nucl. Phys. B352 (1992) 266.

29. K. Jansen, C. Liu, M. Lüscher, H. Simma, S. Sint, R. Sommer, P. Weisz and U. Wolff, Phys. Lett. 372B (1996) 275;

M. Lüscher, S. Sint, R. Sommer and P. Weisz, Nucl. Phys. B478 (1996) 365;

M. Lüscher, S. Sint, R. Sommer, P. Weisz and U. Wolff, Nucl. Phys. B491 (1997) 323.

30. M.K. Gaillard and B.W. Lee, Phys. Rev. Lett. 33 (1974) 108;

G. Altarelli and L. Maiani, Phys. Lett. 52B (1974) 351;

M.A. Shifman, A.I. Vainshtein and V.I. Zakharov, Nucl. Phys. B120 (1977) 316 and Sov. Phys. JEPT 45 (1977) 670;

A.J. Buras and P.H. Weisz, Nucl. Phys. B333 (1990) 66 and Phys. Lett. B192 (1987) 138.

31. A.J. Buras, "Probing the Standard Model of
Particle Interactions", hep-ph/9806471

M. Battaglia et al., hep-ph/0304132.

32. A. Morel, J. Phys. (France) 48 (1987) 1111; S.R. Sharpe and N. Shoresh, Phys. Rev. D64 (2001) 114510.

33. M. Golterman, T. Izubuchi and Y. Shamir, hep-lat/0509190

34. A. Pich and E. de Rafael, Nucl. Phys. B358 (1991) 311;

J. Gasser and U.G. Meissner, Phys. Lett. B258 (1991) 219;

S. Bertolini, J.O. Eeg, M. Fabbrichesi and E.I. Lashin, Nucl. Phys. B514 (1998) 63.

35. L. Maiani and M. Testa, Phys. Lett. B245 (1990) 585.

36. L. Lellouch and M. Lüscher, Commun. Math. Phys. 219 (2001) 31.

37. C.J.D. Lin, G. Martinelli, C.T. Sachrajda and M. Testa, Nucl. Phys. B619 (2001) 467;

C. Kim, C.T. Sachrajda, S.R. Sharpe, Nucl. Phys. B727 (2005) 218 and hep-lat/0510022

38. C. Pena, S. Sint and A. Vladikas, JHEP 0409 (2004) 069; Nucl. Phys. B (Proc. Suppl.) 119 (2003) 368; ibidem 129 (2004) 263 and 140 (2005) 362 .

39. C. Bernard, A. Soni and T. Draper, Phys. Rev. D3 (1987) 3224;

C. Bernard, T. Draper, G. Hockney and A. Soni, Nucl. Phys. B (Proc. Suppl.) 4 (1988) 483.

40. L. Maiani, G. Martinelli, G.C. Rossi and M. Testa, Nucl. Phys. B289 (1987) 505.

41. P. Hernàndez and M. Laine, JHEP 0301 (2003) 063.

42. A.M. Abdel-Rehim, R. Lewis and R.M. Woloshyn, Phys. Rev. D71 (2005) 094505.

43. K. Jansen, M. Papinutto, A. Shindler, C. Urbach and I. Wetzorke, Phys. Lett. B619 (2005) 184; JHEP 0509 (2005) 071 and Proc. Sci. LAT2005:231, 2005.

44. M. Della Morte, R. Frezzotti and J. Heitger, Nucl. Phys. B (Proc. Suppl.) 106 (2002) 260 and hep-lat/0111048

45. V. Lubicz, proceedings of LATTICE2005.

46. S. Weinberg, Phys. Rev. D7 (1973) 1068.

47. W. Bietenholz et al. [ $\chi \mathrm{LF}$ Collaboration], JHEP 0412 (2004) 044.

48. F. Farchioni et al., hep-lat/0509131 